\title{
Práticas Integrativas e Complementares: inserção no contexto do ensino Odontológico
}

\author{
Rodrigo Noll Gonçalves*; Jéssica Rodrigues da Silva Noll Gonçalves**; Marilene da Cruz Magalhães \\ Buffon***; Raquel Rejane Bonato Negrelle****; Guilherme Souza Cavalcanti de Albuquerque***
}

\footnotetext{
* Mestre, Programa de Pós-Graduação em Saúde Coletiva, Universidade Federal do Paraná, Cirurgião-Dentista na Estratégia Saúde da Família de Campo Largo, PR

** Mestranda, Programa de Pós-Graduação em Saúde Coletiva, Universidade Federal do Paraná

*** Docente, Programa de Pós-Graduação em Saúde Coletiva, Universidade Federal do Paraná

**** Docente, Programa de Pós-Graduação em Agronomia, Universidade Federal do Paraná
}

Recebido em 07/08/2017. Aprovado em 08/04/2018.

\begin{abstract}
RESUMO
O presente estudo visa discutir a inserção de Práticas Integrativas e Complementares (PIC) nos modelos flexneriano e giesiano, e seus reflexos na formação superior em Odontologia. Para tal, foi realizado um estudo reflexivo baseado em revisão de literatura. Foram consultadas publicações indexadas nas bases de dados Scientific Electronic Library Online e Biblioteca Virtual em Saúde. Documentos oficiais e periódicos não indexados foram investigados por meio de busca ativa. Os descritores aplicados foram: práticas integrativas e complementares, complementary and integrative practices, relatório flexner, flexner report, relatório gies, gies report, Odontologia de mercado, market dentistry, modelo assistencial em saúde. Observou-se que as características e concepções inseridas nos modelos provenientes dos relatórios Flexner e Gies, de 1910 e 1926 respectivamente, privilegiam a prática dita tecnológica, com a exclusão das práticas tradicionais. As PIC, apesar de seu potencial terapêutico, enfrentam, ainda, resistências de ordem científica, econômica e cultural para sua plena adoção. Nessa lógica, discutem-se os atuais desafios, bem como possibilidades e potencialidades da inserção de PIC na atenção odontológica.
\end{abstract}

Descritores: Terapias Complementares. Medicina Integrativa. Odontologia. Educação Superior.

\section{INTRODUÇÃO}

A integração entre ensino e serviço na área da saúde é entendida como um trabalho coletivo, pactuado e integrado entre universidade e serviços de saúde, a fim de ampliar a qualidade da atenção aos usuários destes serviços, assim 
como oferecer uma formação profissional qualificada ${ }^{1}$. Por conseguinte, faz-se necessária a compreensão, no meio acadêmico, do modelo assistencial, termo polissêmico pertinente à forma de organização dos serviços de saúde e de desenvolvimento das práticas, considerando os valores que orientam o modo como a sociedade define saúde e os direitos dos seres humanos em relação à vida ${ }^{2}$.

Não obstante, as práticas integrativas e complementares (PIC) estão inseridas dentro dos modelos assistenciais. O termo PIC engloba as práticas da Medicina tradicional chinesa, acupuntura, homeopatia, fitoterapia, termalismo/crenoterapia e antroposofia ${ }^{3}$. Estas práticas apresentam ênfase no desenvolvimento do vínculo terapêutico, na integração do ser humano com o meio ambiente e a sociedade, numa visão ampliada do processo saúde-doença e na promoção do cuidado ${ }^{4}$, atuando como coadjuvantes de tratamentos convencionais ${ }^{5}$. Tal fato evidencia a importância da inserção destas práticas nos serviços de saúde e, principalmente, sua abordagem nas instituições de ensino superior na área da saúde.

A adoção de tais práticas, no entanto, enfrenta obstáculos de ordem não somente técnica e científica, mas ideológica e econômica. A conformação das práticas hegemônicas e da formação dos profissionais de saúde é fortemente influenciada por um ideário que desqualifica as iniciativas que se afastam do modelo conhecido como Medicina tecnológica ${ }^{6}$.

Apesar dos grandes avanços alcançados pela Medicina tecnológica, a Organização Mundial da Saúde (OMS) reconhece que grande parte da população dos países em desenvolvimento depende de práticas tradicionais para sua atenção, sendo que $80 \%$ desta população utilizam-nas nos cuidados básicos de saúde, recorrendo, na grande maioria das vezes, a plantas ou preparações destas ${ }^{7}$.
Dentre as PIC aplicadas à Odontologia, destaca-se a fitoterapia, destinada aos estudos dos princípios científicos do cuidado fitoterápico embasados na multidisciplinaridade, inseridos na prática profissional, no resgate do saber popular, no seu uso e aplicabilidade ${ }^{8}$.

A oferta das PIC gera a possibilidade de mudanças no oneroso modelo biomédico, hegemônico na atenção à saúde. Pesquisas sobre o tema possibilitam a informação aos gestores do sistema de saúde sobre a potencialidade destas práticas e a mobilização das equipes de saúde para sua implementação ${ }^{9}$.

$\mathrm{O}$ avanço das PIC pode ser compreendido como expressão de um movimento que se identifica com novos modos de aprender e praticar a saúde, visto que essas práticas se caracterizam pela interdisciplinaridade e por linguagens próprias. Tais práticas se contrapõem à visão altamente tecnológica de saúde que impera na sociedade de mercado, cuja finalidade principal é gerar lucro e fragmentar o tratamento do indivíduo em especialidades que não dão conta da totalidade do ser humano ${ }^{10}$.

Neste contexto, as visões provenientes dos relatórios Flexner e Gies, de 1910 e 1926, respectivamente, caracterizam modelos de atenção que influenciaram a concepção de prática odontológica, ao privilegiar uma visão tecnicista e individualizada ${ }^{11}$. Tais modelos influenciaram ainda o ensino odontológico que, tradicionalmente, foi direcionado a uma prática clínica especializada e biotecnicista ${ }^{12}$, apesar das mudanças ocorridas nas Diretrizes Curriculares Nacionais (DCN) para os cursos de Odontologia ${ }^{13}$.

Nessa lógica, o presente estudo visa discutir a inserção de PIC nos modelos flexneriano e giesiano e seus reflexos na formação superior em Odontologia, os atuais desafios, possibilidades e potencialidades de inserção nos modelos de atenção em saúde 
vigentes no Brasil.

\section{PERCURSO METODOLÓGICO}

Trata-se de um estudo exploratório e reflexivo que busca proporcionar maior familiaridade com o problema, com vistas a torná-lo mais explícito ou a construir hipóteses, tendo como objetivo principal o aprimoramento de ideias ou a descoberta de intuições ${ }^{14}$, baseado na literatura. Marconi e Lakatos (2003) ${ }^{15}$ consideram que a busca de fontes bibliográficas e documentais constitui importante fonte de informações que permite a demonstração de contradições ou a reafirmação de comportamentos e atitudes.

Para tal, foram consultadas as bases de dados Scientific Electronic Library Online (SciELO) e Biblioteca Virtual em Saúde (BVS). Os descritores em ciências da saúde aplicados foram: práticas integrativas e complementares, complementary and integrative practices, relatório flexner, flexner report, relatório gies, gies report, Odontologia de mercado, market dentistry, modelo assistencial em saúde. Foram selecionados artigos científicos relacionados ao tema, publicados entre 1986 e 2017, nos idiomas português e inglês. Documentos oficiais e periódicos não indexados foram investigados por meio de busca ativa.

\section{MODELOS DE ATENÇÃO}

A literatura relacionada ao tema se encontra bastante dispersa, existindo poucos artigos que relacionem os modelos assistenciais às PIC em Odontologia. Buscou-se articular publicações que tratam do assunto em geral, para então realizar uma conexão e discussão entre as PIC e a Odontologia.

Em 1910, foi publicado o estudo Medical Education in the United States and Canada - a report to the Carnegie Foundation for the advancement of teaching, por Abraham
Flexner ${ }^{16}$, mais conhecido como Flexner Report, ou Relatório Flexner. Este documento foi elaborado após a avaliação da situação de diversas escolas médicas dos Estados Unidos da América (EUA), ao constatar que vinha ocorrendo a formação de uma elite profissional. Por conseguinte, recomendou o fechamento de 124 das 155 escolas existentes no país ${ }^{17}$.

Flexner concluiu que nessas 124 escolas médicas, os alunos não tinham preparo prévio adequado, não havia laboratórios nem relação entre formação científica e trabalho clínico, os professores não apresentavam controle sobre os hospitais universitários, os currículos não eram padronizados e o ensino era comercializado ${ }^{17}$.

O relatório Flexner foi responsável pela mais importante reforma das escolas médicas de todos os tempos nos EUA, com profundas implicações para a formação médica e para a Medicina mundial ${ }^{18,19}$. Não obstante, este relatório estendeu-se a outros campos de conhecimento na área da saúde, consolidando a arquitetura curricular que predomina até os dias atuais na rede universitária dos países industrializados ${ }^{20}$. Na prática, o relatório Flexner introduziu critérios de cientificidade e institucionalidade para regulação da formação acadêmica e profissional no campo da saúde ${ }^{21}$.

A formação do cirurgião-dentista também sofreu influência direta deste relatório. Segundo Pereira et al. $(2003)^{11}$, as raízes históricas, culturais e sociais na formação do cirurgiãodentista pautada no modelo flexneriano ou de Medicina científica, gerou uma prática individualista, curativa, tecnicista, especializada e biologicista. De acordo com o relatório Flexner, não há lugar para as práticas ou terapias complementares, como homeopatia, fitoterapia, acupuntura, entre outras, pelo fato de não se enquadrarem no paradigma científico ${ }^{22}$.

Por conseguinte, verifica-se um paradoxo, ao considerar que os profissionais da saúde bucal 
vêm secularmente sendo formados de acordo com o modelo educacional biomédico e flexneriano, não apresentando perfil adequado para atuar no modelo de saúde vigente ${ }^{12}$. Segundo Lima Júnior $(2005)^{22}$, a consequência foi um perfil profissional com prática orientada para atividades curativistas, individualistas, fragmentadas e centradas na clínica privada, sem lugar para o desenvolvimento de ações coletivas ligadas ao campo da atenção básica de saúde.

De acordo com Oliveira et al. $(2008)^{23}$, no processo de formação dos profissionais de saúde, observa-se que as disciplinas ligadas ao social se encontram em um plano secundário, devido à influência da concepção flexneriana, que privilegia o biologicismo, o tecnicismo, o individualismo, o mecanicismo e a formação voltada para a doença.

Nesse sentido, nota-se a presença de uma dívida histórica no campo da Odontologia para com seus profissionais, dado que a formação odontológica não prioriza as disciplinas ou os saberes científicos relacionados ao social. Durante cerca de $90 \%$ do tempo dedicado à sua formação, o graduando aprende a lidar ou tratar a doença em detrimento da saúde ${ }^{23}$.

Após a publicação do relatório Flexner, a Fundação Carnegie decidiu realizar um trabalho similar para a educação odontológica, por meio de um levantamento sobre o ensino em Odontologia, nos EUA e no Canadá ${ }^{24}$.

Em 1926, foi publicado o estudo Dental Education in the United States and Canada $-a$ report to the Carnegie Foundation for the advancement of teaching, elaborado por William J. Gies, mais conhecido como Gies Report, ou Relatório Gies ${ }^{25}$. O relatório Gies é considerado atualmente a revisão mais abrangente e influente da educação odontológica no contexto de uma profissão $^{24}$.

Dentre os efeitos das recomendações do relatório Gies, visíveis ao longo do século XX, destacam-se a ênfase nas ciências biológicas e clínicas; progressiva agregação tecnológica com especialização precoce; práticas curativocirúrgicas e reabilitadoras adotadas como modelo de intervenção preferencial sobre indivíduos e doenças, elegendo o mercado privado como lócus privilegiado do exercício profissional ${ }^{26}$.

A essência da Odontologia de mercado, fundada na lógica de Gies, está na base biológica e individual de atuação clínica, e em sua organicidade ao modo de produção capitalista, por meio da transformação dos cuidados de saúde em mercadorias, impondo-lhes deformações mercantilistas e éticas ${ }^{27}$. No entanto, é importante ressaltar que o relatório Gies baseouse nos elementos ideológicos apresentados no relatório Flexner. Por conseguinte, ambos são caracterizados por mecanicismo, biologicismo, assistência individual, especialização, tecnificação do ato médico-odontológico, exclusão de práticas tradicionais, além de ênfase em Medicina e Odontologia curativa ${ }^{28}$.

A Odontologia 'flexneriana', entendida até então como a prática de universalidade biológica, está orientada para a cura ou alívio das doenças, ou restauração de lesões. Com enfoque no individual de seu objeto, pela concepção mecanicista do homem, pela apropriação do conhecimento em tecnologia de alta densidade de capital, pela dominância da especialização, pela seletividade de sua clientela e pela exclusão de formas alternativas de prática odontológica ${ }^{29}$.

Para Albuquerque et al. $(2008)^{30}$, o modelo de atenção à saúde predominantemente vigente, estava 'centrado no procedimento', pelo fato de que o principal compromisso do ato de assistir à saúde era por meio da produção de procedimentos, e de forma secundária, às necessidades dos usuários. Neste contexto, a forma de atenção à saúde na época priorizava $o$ procedimento, desconsiderando em muitos casos o motivo principal da procura do serviço. No 
entanto, esta concepção de prática mercadológica continua inserida nos currículos de formação superior em Odontologia.

De acordo com Toassi et al. (2012) ${ }^{17}$, a formação odontológica no Brasil ainda sofre influência do modelo 'flexneriano', proveniente dos elementos do relatório Flexner, desconsiderando o pluralismo diagnósticoterapêutico que as PIC promovem ${ }^{31}$.

Segundo Narvai $(2006)^{27}$, a Odontologia de mercado jamais perdeu a hegemonia no sistema de saúde brasileiro, e tal concepção não apenas predomina no setor privado, como segue exercendo grande influência sobre os serviços públicos. Todavia, ao exercer ainda forte influência no desenvolvimento da ciência e da tecnologia, a Odontologia de mercado parece não responder bem aos problemas de saúde bucal da população, devido ao seu caráter essencialmente curativo, de custo elevado e baixa cobertura populacional $^{17}$.

A necessidade de mudança da prática odontológica tem sido apresentada reiteradamente por diversos autores. Segundo Mendes $(1986)^{29}$, já no contexto das pretensões para instaurar a Reforma Sanitária, haveria a necessidade de mudar a prática odontológica hegemônica, 'flexneriana', por um modelo de Odontologia integral. Cordón (1997) ${ }^{32}$ afirma que a prática odontológica deveria mudar do modelo de Odontologia integral, com forte inclinação 'giesiana' na formação acadêmica, para um modelo com abertura de espaços sociais em busca de uma Odontologia inserida integralmente na saúde, abordando de forma participante os espaços sociais.

Ainda, segundo Cordón (1997) $)^{32}$, vida saudável para todos significa universalidade no acesso à atenção à saúde e a uma qualidade de vida melhor, mais solidária, equitativa e justa. Dessa forma, qualquer proposta de intervenção em saúde deve apreender a realidade, em cada espaço social, para poder compreender o indivíduo, o cidadão e as redes sociais que se constroem, podendo, desta forma, definir suas necessidades, seus problemas e identificar os processos coletivos necessários de transformação do modo de vida em sociedade.

O modelo hegemônico de assistência odontológica foi caracterizado, no relatório final da VII Conferência Nacional de Saúde, como ineficaz, ineficiente, descoordenado, de baixa cobertura e alta complexidade, com enfoque curativo, de caráter mercantilista e monopolista, para o qual contribui a inadequação no preparo dos recursos humanos ${ }^{33,34}$.

A necessidade de revisão da formação odontológica tem sido apresentada com ênfase especial a partir do início do século 21. O artigo $3^{\circ}$ da Resolução CNE/CES 3, de 19 de fevereiro de 2002, que institui as DCN para os cursos de graduação em Odontologia, determina o "perfil do formando egresso/profissional o CirurgiãoDentista, com formação generalista, humanista, crítica e reflexiva, para atuar em todos os níveis de atenção à saúde, com base no rigor técnico e científico. Capacitado ao exercício de atividades referentes à saúde bucal da população, pautado em princípios éticos, legais e na compreensão da realidade social, cultural e econômica do seu meio, dirigindo sua atuação para a transformação da realidade em benefício da sociedade" 13 .

Neste sentido, a legislação consubstancia uma vontade de mudança para o ensino superior no Brasil, e para a Odontologia em particular ${ }^{28}$. Se, como enunciam as DCN, a formação do cirurgião-dentista será generalista, humanista, crítica e reflexiva, visando a transformação da realidade a partir de sua compreensão, impõe-se uma profunda reflexão pedagógica.

Segundo Moysés $\left(2003\right.$, p.87) ${ }^{28}$, “[...], a partir de uma epistemologia voltada para atividades pedagógicas humanistas, podem-se criar processos socialmente relevantes e com 
sujeitos capazes de formular a crítica a modelos hegemônicos, exclusivamente voltados para atender o mercado". A humanização das práticas pedagógicas pressupõe, ainda, a criação de processos educativos socialmente relevantes, além da crítica ao modelo de formação mecanicista e de tecnificação da prática profissional, voltada ao atendimento das demandas do mercado ${ }^{35}$.

\section{PRÁTICAS INTEGRATIVAS E COMPLE- MENTARES}

A alta demanda pelas PIC pode representar avanços no movimento cultural, que inclui essas práticas para além de uma forma de tratar o adoecimento. Portanto, podem ser acessadas pela população, como um direito de cidadania ${ }^{36}$.

As PIC constituem-se de procedimentos e medicamentos de baixo custo, possuem pouca potencialidade lucrativa e demandam um esforço adicional do profissional para o estabelecimento do diagnóstico ou de algum procedimento terapêutico, conflitando com a lógica da gestão focada na eficiência e na eficácia da acumulação flexível. Outrossim, podem estar associadas a todos os níveis de assistência, inclusive na atenção terciária. Porém, apesar de terem sido definidas como eixo importante para a promoção do cuidado em saúde e com a finalidade de expansão da lógica dos serviços da atenção básica, ainda promovem resistências entre gestores e profissionais ${ }^{37}$.

Em 1991, a OMS reiterou a importante contribuição da Medicina tradicional na prestação da assistência e solicitou aos estados-membros que intensificassem a cooperação entre praticantes da Medicina tradicional e da assistência sanitária moderna, especialmente quanto ao emprego de medicamentos tradicionais de eficácia científica demonstrada, contribuindo para a viabilidade econômica da atenção médica ${ }^{7}$. Entretanto, apesar das diversas contribuições que a Medicina tradicional pode oferecer, os modelos hegemônicos, de base flexneriana e giesiana, persistem em desconsiderá-la, afirmando sua ineficácia.

No entanto, o campo das PIC em saúde constitui um fenômeno crescente no Brasil contemporâneo, visto que tais recursos têm sido apropriados e difundidos, além dos serviços públicos de saúde, por clínicas particulares, comunidades tradicionais, igrejas, movimentos sociais e entidades não governamentais ${ }^{31}$.

No final da década de 1970, a OMS criou o Programa de Medicina Tradicional, objetivando a formulação de políticas públicas na área. No Brasil, a legitimação e institucionalização dessas abordagens de atenção à saúde tiveram início a partir da década de 1980, após a criação do Sistema Único de Saúde (SUS) ${ }^{38}$. A implantação da Política Nacional de Práticas Integrativas e Complementares (PNPIC) permitiu, então, que no ano de 2006, algumas PIC fossem incorporadas ao SUS $^{39}$.

No que diz respeito à assistência odontológica, já existem políticas públicas de incorporação das PIC, e o próprio Conselho Federal de Odontologia (CFO), por meio da resolução $\mathrm{n}^{\mathrm{o}} 82$, de 25 de setembro de 2008, reconhece e regulamenta o uso das mesmas pelo cirurgião-dentista $^{8}$. Ainda, a Resolução ${ }^{\circ}{ }^{\circ}$ 165, 24 de novembro de 2015, reconhece e regulamenta o uso pelo cirurgião-dentista da prática integrativa e complementar à saúde bucal Odontologia antroposófica $^{40}$. Tais iniciativas enfatizam a importância do uso destas práticas, inseridas na Odontologia, em vista de seus resultados positivos sobre o processo saúde-doença.

Neste sentido, o reconhecimento das PIC em saúde bucal é relativamente recente. De acordo com os critérios de busca, alguns estudos relacionados à fitoterapia e Odontologia ${ }^{41,42}$ foram identificados. Porém, segundo Reis et al. $(2014)^{41}$, são escassos os estudos relacionados ao tema em Odontologia. Ainda, de acordo com Evangelista et 
al. $(2013)^{42}$, são poucos os relatos de utilização de substâncias naturais em Odontologia, o que justifica a necessidade de maiores estudos, além de capacitação dos profissionais, a fim de garantir a aplicação das PIC de maneira eficaz e segura.

Gonçalo e Barros $(2014)^{43}$ realizaram uma revisão sistemática na qual foram analisadas evidências positivas e negativas relativas ao uso das PIC em saúde bucal. Foram constatados mais resultados positivos relacionados à fitoterapia, dentre as PIC analisadas. Os autores relataram ainda a existência de muitos obstáculos para se pesquisar, analisar e estabelecer a validade e a confiabilidade dos resultados das PIC em Odontologia.

\section{CONSIDERAÇÕES FINAIS}

A superação parcial das resistências científicas, econômicas e culturais, permitiu a aceitação e a implantação legal das PIC no sistema de saúde brasileiro. Persiste, no entanto, o cenário de disputa com os modelos de atenção fundados nas concepções de Flexner e Gies, nos quais tais práticas são desqualificadas. Resta, ainda, longo caminho na formação de profissionais capacitados para desenvolvê-las e, efetivamente, legitimá-las.

É essencial, portanto, que ocorra o desenvolvimento do processo de mudança curricular na formação superior em Odontologia para o desempenho de tais práticas, tornando sua institucionalização mais consistente, segura e eficaz, tanto na atenção à saúde em âmbito público, quanto privado, permitindo à população brasileira beneficiar-se das PIC como alternativas eficazes e de baixo custo para os cuidados em saúde.

\section{ABSTRACT \\ Complementary and Integrative Practices: Incorporation into Dental Education}

The present study aims to discuss the inclusion of complementary and integrative practices (CIP) in the Flexnerian and Giesian models and their effects on higher education in dentistry. Thus, a reflexive study based on a literature review was carried out. Indexed publications were consulted in the databases Scientific Electronic Library Online and Virtual Health Library. Official documents and non-indexed journals were searched. The descriptors applied were as follows: práticas integrativas e complementares, complementary and integrative practices, relatório flexner, flexner report, relatório gies, gies report, Odontologia de mercado, market dentistry, modelo assistencial em saúde. It was observed that the features and concepts embedded in the models from the Flexner and Gies reports, dated 1910 and 1926, respectively, favor technological practices and exclude traditional practices. Despite their therapeutic potential, CIP also face scientific, economic and cultural resistance to their full adoption. By this logic, the current challenges as well as the possibilities and potential of CIP in dental care are discussed.

Descriptors: Complementary Therapies. Integrative Medicine. Dentistry. Education, Higher.

\section{REFERÊNCIAS}

1. Faé JM, Silva Júnior MF, De Carvalho RB, Esposti CDD, Pacheco KTS. A integração ensino-serviço em Odontologia no Brasil. Rev ABENO. 2016;16(3):7-18.

2. Fertonani HP, Pires DEP, Biff D, Scherer MDA. Modelo assistencial em saúde: conceitos e desafios para a atenção básica brasileira. Ciênc Saúde Coletiva. 2015;20(6):1869-78.

3. Gontijo MBA, Nunes MF. Práticas Integrativas e Complementares: conhecimento e credibilidade de profissionais do serviço público de saúde. Trab Educ Saúde. 2017;15(1):301-20. 
4. Brasil. Ministério da Saúde. Gabinete do Ministro. Portaria n ${ }^{\circ}$ 971, de 3 maio de 2006. Aprova a Política Nacional de Práticas Integrativas e Complementares (PNPIC) no Sistema Único de Saúde. Diário Oficial da União, Brasília, DF. 2006;(84):20-25. Seção 1. [Acesso em 16 out. 2016]. Disponível em: http://bvsms.saude.gov.br/bvs/saude legis/gm/2006/prt0971_03_05_2006.html.

5. Ischkanian PC, Pelicioni MCF. Challenges of complementary and medicine in the SUS aiming to health promotion. J Human Growth Develop. 2012;22(2):233-8.

6. Schraiber LB. O médico e seu trabalho. Limites da liberdade. São Paulo: Hucitec; 1993. 229 p.

7. Brasil. Ministério da Saúde. Secretaria de Ciência, Tecnologia e Insumos Estratégicos. Departamento de Assistência Farmacêutica. Política nacional de plantas medicinais e fitoterápicos. Brasília: Ministério da Saúde, 2006. 60 p. - (Série B. Textos Básicos de Saúde).

8. Brasil. Conselho Federal de Odontologia. Resolução no 82, de 25 de setembro de 2008. Reconhece e regulamenta o uso pelo cirurgião-dentista de práticas integrativas e complementares à saúde bucal. Diário Oficial da União, Seção 1. Brasília, DF. 2008;(190):105-107.

9. Ibiapina WV, Leitão BP, Batista MM, Pinto DS. Inserção da fitoterapia na Atenção Primária aos usuários do SUS. Rev Ciênc Saúde Nova Esperança. 2014;12(1):58-68.

10. Telesi Júnior E. Práticas integrativas e complementares em saúde, uma nova eficácia para o SUS. Rev Estudos Avançados. 2016;30(86):99-112.

11. Pereira DQ, Pereira JCM, Assis MMA. A prática odontológica em Unidades Básicas de Saúde em Feira de Santana (BA) no processo de municipalização da saúde: individual, curativa, autônoma e tecnicista. Ciênc Saúde Coletiva. 2003; 8(2):599-609.

12. Emmerich A, Castiel LD. A ciência odontológica, Sísifo e o "efeito camaleão". Interface (Botucatu). 2009; 13(29):339-51.

13. Brasil. Conselho Nacional de Educação. Resolução $\mathrm{N}^{\circ}$ CNE/CNS 3, de 19 de fevereiro de 2002. Institui Diretrizes Curriculares Nacionais do Curso de Graduação em Odontologia. Diário Oficial da União. Seção 1. Brasília, DF. 2002;(42):10-11. [Acesso em 9 jun. 2017]. Disponível em: http://pesquisa.in.gov.br/ imprensa/jsp/visualiza/index.jsp?data $=04 / 0$ $\underline{3 / 2002 \& \text { jornal }=1 \& \text { pagina }=10 \& \text { totalArquiv }}$ $\underline{\text { os }=120}$

14. Gil AC. Como elaborar projetos de pesquisa. 4. ed. São Paulo: Atlas; 2002. 192 p.

15. Marconi MA, Lakatos EM. Fundamentos de metodologia científica. 5. ed. São Paulo: Atlas; 2003. $311 \mathrm{p}$.

16. Flexner A. Medical Education in the United States and Canada. New York: The Carnegie Foundation for The Advancement of Teaching, 1910. (Bulletin, 4).

17. Toassi RFC, Stobäus CD, Mosquera JJM, Moysés SJ. Integrated curriculum for teaching dentistry: new directions for training in the field of healthcare. Interface (Botucatu). 2012;16(41):529-42.

18. Pagliosa FL, Da Ros MA. O relatório Flexner: para o bem e para o mal. Rev Bras Educ Med. 2008;32(4):492-99.

19. Ludmerer KM. Understanding the Flexner Report. Acad Med. 2010;85(2):193-6.

20. Hora DL, Erthal RMC, Souza CTV, Hora EL. Propostas inovadoras na formação do profissional para o Sistema Único de Saúde. Trab Educ Saúde. 2013;11(3):471-86.

21. Almeida Filho N. Reconhecer Flexner: inquérito sobre produção de mitos na educação médica no Brasil contemporâneo. 
Cad Saúde Pública. 2010;26(12):2234-49.

22. Lima Júnior JF. Perspectivas dos cirurgiõesdentistas sobre a inserção da fitoterapia na atenção básica de saúde [dissertação]. Natal: Universidade Federal do Rio Grande do Norte; 2005.

23. Oliveira ET, Lima Júnior JF, Soares FNCS, Maia ER. A odontologia social no contexto da promoção da saúde. Rev Bras Promoç Saúde. 2008;21(1):75-9.

24. Dunoff RB. It is time for a new Gies report. J Dent Educ. 2006;70(8):809-19.

25. Gies WJ. Dental education in the United States and Canada. New York: The Carnegie Foundation for The Advancement of Teaching, 1926. (Bulletin, 19).

26. Moysés SJ. Políticas de atenção à saúde bucal: o cenário internacional e o Brasil. In: Giovanella, L.; Escorel, S.; Lobato, L.V.C.; Noronha, J.C.; Carvalho, A.I. Políticas e Sistema de Saúde no Brasil. 2. ed. Rio de Janeiro: FIOCRUZ; 2012. p.609-633.

27. Narvai PC. Saúde bucal coletiva: caminhos da odontologia sanitária à bucalidade. Rev Saúde Pública. 2006;40(N Esp):141-147.

28. Moysés SJ. A humanização da educação em odontologia. Rev Pro-Posições. 2003;14(1):87-106.

29. Mendes EV. A Reforma Sanitária e a Educação Odontológica. Cad Saúde Pública. 1986;2(4):533-52.

30. Albuquerque VS, Gomes AP, Rezende CHA, Sampaio MX, Dias OV, Lugarinho RM. A integração ensino-serviço no contexto dos processos de mudança na formação superior dos profissionais da saúde. Rev Bras Educ Méd. 2008;32(3):35662.

31. Andrade JT, Da Costa LFA. Medicina Complementar no SUS: práticas integrativas sob a luz da Antropologia médica. Rev Saúde Soc. 2010;19(3):497-508.
32. Cordón J. A construção de uma agenda para a saúde bucal coletiva. Cad Saúde Públ. 1997;13(3):557-63.

33. Brasil. Conferência Nacional de Saúde, 7. Centro de Documentação do Ministério da Saúde. Anais da $7^{\text {a }}$ Conferência Nacional de Saúde. Extensão das ações de saúde através dos serviços básicos. Brasília, DF. 1980;280p.

34. Canalli CSE, Gonçalves SS, Chevitarese L, Silveira RG, Miasato JM. A humanização na Odontologia: uma reflexão sobre a prática educativa. Rev Bras Odontol. 2011;68(1):44-8.

35. Moysés ST, Moysés SJ, Kriger L, Schmitt EJ. Humanizando a educação em Odontologia. Rev ABENO. 2003;3(1):5864.

36. Lima KMSV, Silva KL, Tesser CD. Práticas integrativas e complementares e relação com promoção da saúde: experiência de um serviço municipal de saúde. Interface (Botucatu). 2014;18(49):261-72.

37. Sacramento HT, Gentilli RML. Mundialização do capital e política de saúde: desafios para as práticas integrativas e complementares no SUS. Rev Pol Públ. 2016;20(1):103-20.

38. Brasil. Ministério da Saúde. Secretaria de Atenção à Saúde. Departamento de Atenção Básica. Política Nacional de Práticas Integrativas e Complementares no SUS: atitude de ampliação de acesso. 2. ed. Brasília: Ministério da Saúde; 2015. 96 p.

39. Brasil. Ministério da Saúde. Secretaria de Ciência, Tecnologia e Insumos Estratégicos. Departamento de Assistência Farmacêutica e Insumos Estratégicos. Programa Nacional de Plantas Medicinais e Fitoterápicos. Brasília: Ministério da Saúde; 2009. 136 p.

40. CRO-GO. Conselho Regional de Odontologia de Goiás. Resoluções do CFO 
reconhecem e normatizam práticas importantes da Odontologia. [Acesso em 07 nov. 2017]. Disponível em: http://www. crogo.org.br/index.php/noticias/514-resolucoe s-do-cfo-reconhecem-e-normatizam-praticasimportantes-da-odontologia

41. Reis LBM, Farias AL, Bollella AP, Silva HKM, Canuto MIC, Zambelli JC, et al. Conhecimentos, atitudes e práticas de Cirurgiões-Dentistas de Anápolis-GO sobre a fitoterapia em odontologia. Rev Odontol UNESP. 2014;43(5):319-25.
42. Evangelista SS, Sampaio FC, Parente RC, Bandeira MFCL. Fitoterápicos na odontologia: estudo etnobotânico na cidade de Manaus. Rev Bras Pl Med. 2013;15(4):513-9.

43. Gonçalo CS, Barros NF. The use of complementary and integrative practices in oral health. Rev Acta Scientiarum. 2014;36(2):281-91.

\section{Correspondência para:}

Rodrigo Noll Gonçalves

e-mail: rodrigo.noll@hotmail.com

Programa de Pós-Graduação em Saúde Coletiva Universidade Federal do Paraná

Rua Padre Camargo, $280-5^{\circ}$. andar Alto da Glória 80060-240 Curitiba, PR 